\title{
Divergence-constrained Moving Least Squares for Fluid Simulation
}

\author{
Jeong-Mo Hong \\ Korea University \\ saden@korea.ac.kr
}

\author{
Jong-Chul Yoon \\ Korea University \\ alskay2k@korea.ac.kr
}

\author{
Chang-Hun Kim \\ Korea University \\ chkim@korea.ac.kr
}

\begin{abstract}
Developing suitable interpolation methods to simulate dynamic motions of continuous materials such as fluids is an important problem. In this paper, we propose a novel method to enforce the divergence condition to the interpolated velocity field by moving least squares, by means of the diffusive derivatives and moving divergence constraints that allow the practical use and easy implementation. As results, we present the velocity interpolation examples and a fluid-like particle simulation method to show the meaningful potential of our method as a tool of physical interpolation for fluid simulations.
\end{abstract}

Keywords: Fluid simulation, moving least squares, vector interpolation, divergence constraint, computer animation

\section{Introduction}

Due to the digital nature of computing machineries, data should be stored and processed after being discretely sampled. Therefore, it is very important to develop suitable interpolation methods, especially when we want to simulate dynamic motions of continuous materials, because we need to retrieve data from between the sampling points frequently.

Fluid simulation has become a very popular topic to study in computer graphics. One direction of research is to improve the simulation accuracy by incorporating higher-order numerical methods to improve simulation accuracy. Some researchers have tried to improve
semi-Lagrangian advection (initially introduced by Stam [1] to computer graphics) by replacing linear interpolation with higher-order schemes such as the BFECC [2] and CIP [3] methods. These techniques appear to be successful on examples. The hybrid methods of Lagrangian and Eulerian approaches such as Kim et al. [4], Losasso et al. [5], and Hong et al. [6], also require better interpolation techniques, to improve the coupling of grid-based simulators to particles.

Feldman et al. [7] constrain a moving least squares (MLS) approximation with the normal component of velocity in order to obtain a smooth vector field from an adaptive (or hybrid) grid. MLS also looks to be a promising technique for higher-order interpolation. But there is a more challenging question: is it possible to use MLS to enforce physical constraints as well as continuity conditions? That is the motivation of this work.

We propose a novel way of enforcing the divergence condition in an interpolated velocity field using MLS. Figure 1 shows how this approach differs from simple vector interpolation using MLS. Since there must be no divergence in a model of an incompressibility of fluids, our method produces a more physical interpolation of a velocity field without requiring the support of a fluid dynamics solver such as the widely used pressure projection method.

Practical implementation of an MLS approach using diffusive derivatives and the moving divergence constraint turns out to be relatively straightforward. We present ex- 


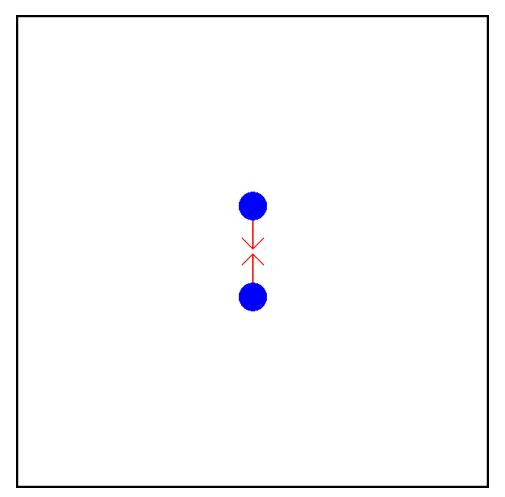

(a) Velocity Constraints

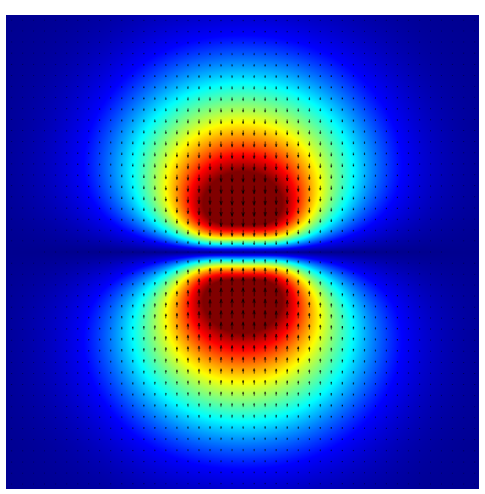

(b) Naïve MLS

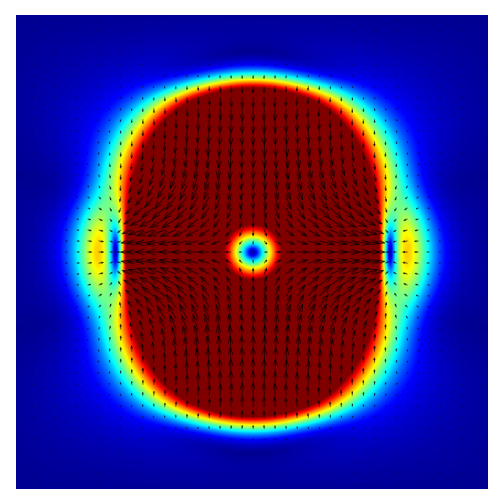

(c) Our MLS

Figure 1: Comparing standard MLS vector interpolation to our pseudo-divergence-free vector interpolation. The colors, from red through green to blue, represent interpolated vectors of increasing magnitude. Our method produces the interpolated vectors with the non-zero horizontal components required to approximate a divergence-free velocity field, even though the given constraints only have vertical components.

amples of divergence-free vector interpolation, divergence-controlled interpolation, and divergence-free extrapolation. We also present a simple method of particle animation that approximates fluid motions without any support from fluids solver. This shows that our method has the potential to improve the physical interpolation component of existing fluid simulation techniques.

\subsection{Our Contributions}

The summary of our contributions to computer graphics community in this work is as follows:

- Demonstrating the potential of MLS for physical interpolation in fluid simulations.

- Introducing pseudo-divergence-free (PDF) MLS, which is an element-free Galerkin method.

- Presenting a simpler and better constraining method than PDF.

- Proposing a fluid-like particle animation technique based on an interpolation method instead of a fluids solver.

\subsection{Related Work}

MLS is a very popular tool for approximating and interpolating scattered data in many areas, and it is not possible to list all of its applications. These include geometric problems (see Shen et al. [8] and its references for examples), and meshless methods [9]. Müller et al. [10] used MLS to compute the spatial derivatives of a point-based surface to model physical deformations, and Feldman et al. [7] used MLS to interpolate fluid velocities.

\section{Moving Least Squares}

We will now introduce the general concept of MLS using an example of scalar interpolation (or approximation), which we will extend to vector interpolations. We will then develop our divergence constrained vector interpolation in Section 3. The easiest introduction to MLS is possibly Nealen [11], and most of the notation that we use comes from Feldman et al. [7] and Shen et al. [8].

\subsection{Overview}

Given $N$ control points located at positions $\mathbf{p}_{i}$ in $\mathbb{R}^{d}$, where $i \ni[1, \ldots, N]$, we wish to find a polynomial function

$$
f(\mathbf{x})=\mathbf{b}^{T}(\mathbf{x}) \mathbf{c}
$$

that interpolates (or closely approximates) the values $\phi_{i}$ at those points as follows:

$$
f\left(\mathbf{p}_{i}\right)=\mathbf{b}^{T}\left(\mathbf{p}_{i}\right) \mathbf{c}=\phi_{i},
$$


where the sampling point $\mathbf{x}$ is $[x, y]$ in $2 \mathrm{D}$ and $[x, y, z]$ in $3 \mathrm{D}, \mathbf{b}(\mathbf{x})=\left[b_{1}(\mathbf{x}), \ldots, b_{k}(\mathbf{x})\right]$ is the basis function of the fitting polynomial, and $\mathbf{c}=$ $\left[c_{1}, \ldots, c_{k}\right]$ is the unknown vector of coefficients. In general, the number of elements in $\mathbf{b}(\mathbf{x})$ (and therefore in $\mathbf{c})$ is given by $k=\frac{(d+m) !}{m ! d !}$, where $f$ is taken from $\Pi_{m}^{d}$, which is the space of polynomials with a total degree of $m$ and $d$ spatial dimensions. In 2D $(d=2)$, we can write

$$
\begin{aligned}
& \mathbf{b}(\mathbf{x})=[1] \text { if } m=0 \\
& \mathbf{b}(\mathbf{x})=[1, x, y] \text { if } m=1 \\
& \mathbf{b}(\mathbf{x})=\left[1, x, y, x^{2}, y^{2}, x y\right] \text { if } m=2
\end{aligned}
$$

and in 3D $(d=3)$, we can write

$$
\begin{aligned}
& \mathbf{b}(\mathbf{x})=[1] \text { if } m=0 \\
& \mathbf{b}(\mathbf{x})=[1, x, y, z] \text { if } m=1 \\
& \mathbf{b}(\mathbf{x})=\left[1, x, y, z, x^{2}, y^{2}, z^{2}, x y, y z, x z\right] \text { if } m=2 .
\end{aligned}
$$

The moving least squares fit is achieved by multiplying all the value constraints described in Equation (2) by the weight functions $\omega\left(\left|\mathbf{r}_{i}\right|\right)$, where $\mathbf{r}_{i}=\mathbf{p}_{i}-\mathbf{x}$, and then gathering them together into one equation, as follows:

$$
\left[\begin{array}{c}
\omega\left(\left|\mathbf{r}_{1}\right|\right) \\
\ddots \\
\omega\left(\left|\mathbf{r}_{N}\right|\right)
\end{array}\right]\left[\begin{array}{c}
\mathbf{b}^{T}\left(\mathbf{p}_{1}\right) \\
\vdots \\
\mathbf{b}^{T}\left(\mathbf{p}_{N}\right)
\end{array}\right] \mathbf{c}=\left[\begin{array}{c}
\omega\left(\left|\mathbf{r}_{1}\right|\right) \\
\ddots \\
\omega\left(\left|\mathbf{r}_{N}\right|\right)
\end{array}\right]\left[\begin{array}{c}
\phi_{1} \\
\vdots \\
\phi_{N}
\end{array}\right] .
$$

This can be abbreviated to

$$
\mathbf{W}(\mathbf{x}) \mathbf{B c}(\mathbf{x})=\mathbf{W}(\mathbf{x}) \Phi,
$$

where $\mathbf{W}$ is a $N \times N$ diagonal matrix, $\mathbf{B}$ is a $N \times k$ matrix, and $\Phi$ is a vector in $\mathbb{R}^{N}$. If we apply the method of normal equations to this equation, we have

$$
\mathbf{B}^{T}(\mathbf{W}(\mathbf{x}))^{2} \mathbf{B c}(\mathbf{x})=\mathbf{B}^{T}(\mathbf{W}(\mathbf{x}))^{2} \Phi .
$$

We can obtain $\mathbf{c}(\mathbf{x})$ by solving this equation as a linear system. Then $f$ at position $\mathbf{x}$ can be determined from Equation (1). We used Cholesky decomposition as the numerical solution method in all examples.

\subsection{Vector Interpolation}

We now extend the scalar interpolation explained in the previous section to a vector field $\mathbf{u}=[u, v, w]^{T}$. By simply substituting each element of $\mathbf{u}$ for $f$ in Equation (2), we obtain the constraint equations:

$\mathbf{u}\left(\mathbf{p}_{i}\right)=\left[\begin{array}{c}u\left(\mathbf{p}_{i}\right) \\ v\left(\mathbf{p}_{i}\right) \\ w\left(\mathbf{p}_{i}\right)\end{array}\right]=\left[\begin{array}{l}\mathbf{b}^{T}\left(\mathbf{p}_{i}\right) \mathbf{c}_{x} \\ \mathbf{b}^{T}\left(\mathbf{p}_{i}\right) \mathbf{c}_{y} \\ \mathbf{b}^{T}\left(\mathbf{p}_{i}\right) \mathbf{c}_{z}\end{array}\right]=\left[\begin{array}{c}u_{i} \\ v_{i} \\ w_{i}\end{array}\right]=\mathbf{u}_{i}$.

In general, vector interpolation can be performed by interpolating each element of the vectors separately. However, we need to formulate a single vector interpolation so that the elements can constrain each other and meet the divergence constraint discussed in Section 3. We therefore rewrite Equation (3) to interpolate vectors as

$$
\mathbf{W}_{v e c}(\mathbf{x}) \mathbf{B}_{v e c} \mathbf{c}_{v e c}(\mathbf{x})=\mathbf{W}_{v e c}(\mathbf{x}) \Phi_{v e c},
$$

where

$$
\mathbf{W}_{v e c}=\left[\begin{array}{c}
\omega\left(\left|\mathbf{r}_{1}\right|\right) \\
\omega\left(\left|\mathbf{r}_{1}\right|\right) \\
\omega\left(\left|\mathbf{r}_{1}\right|\right) \\
\ddots \\
\omega\left(\left|\mathbf{r}_{N}\right|\right) \\
\omega\left(\left|\mathbf{r}_{N}\right|\right) \\
\omega\left(\left|\mathbf{r}_{N}\right|\right)
\end{array}\right]
$$

$$
\mathbf{B}_{v e c}=\left[\begin{array}{cccc}
\mathbf{b}\left(\mathbf{p}_{1}\right) & \mathbf{0} & \mathbf{0} \\
\mathbf{0} & \mathbf{b}\left(\mathbf{p}_{1}\right) & \mathbf{0} \\
\mathbf{0} & \mathbf{0} & \mathbf{b}\left(\mathbf{p}_{1}\right) \\
\vdots & \\
\mathbf{b}\left(\mathbf{p}_{N}\right) & \mathbf{0} & \mathbf{0} \\
\mathbf{0} & \mathbf{b} & \left.\mathbf{p}_{N}\right) & \mathbf{0} \\
\mathbf{0} & \mathbf{0} & \mathbf{b}\left(\mathbf{p}_{N}\right)
\end{array}\right],
$$

$\mathbf{c}_{v e c}=\left[\mathbf{c}_{x}, \mathbf{c}_{y}, \mathbf{c}_{z}\right]$ and $\Phi_{\text {vec }}=\left[\mathbf{u}_{1}, \cdots, \mathbf{u}_{N}\right] . \mathbf{W}_{\text {vec }}$ is a diagonal $d N \times d N$ matrix, $\mathbf{B}_{v e c}$ is a $d N \times d k$ matrix, and $\Phi$ is a vector in $\mathbb{R}^{d N}$. We can perform vector interpolation by solving Equation (6) in the same way that we solved Equation (3) for scalar interpolation in Section 2.1.

\section{Divergence-constrained MLS}

In this section, we introduce the diffusive derivatives and then use them to develop the divergence constraint for MLS.

\subsection{Diffusive Derivatives}

The accurate derivative of $f$ is obtained by differentiating Equation (1), which yields

$$
\frac{\partial f}{\partial \mathbf{x}}=\frac{\partial \mathbf{b}^{T}}{\partial \mathbf{x}} \mathbf{c}+\mathbf{b}^{T} \frac{\partial \mathbf{c}}{\partial \mathbf{x}} .
$$


It is difficult to use the second term on the righthand side of this equation in constraining an MLS formulation although it can be evaluated straightforwardly.

Instead of the accurate derivatives of Equation (7), we introduce the diffuse derivatives

$$
\frac{\partial f}{\partial \mathbf{x}} \simeq \frac{\partial \mathbf{b}^{T}}{\partial \mathbf{x}} \mathbf{c}
$$

proposed by Nayroles et al. [12]. Huerta et al. [13] proved that the diffusive derivatives asymptotically approach the accurate derivatives as the distances between constraint points decrease. More precisely, the diffuse derivatives are identical to the accurate derivatives at constraint points, but there is an error which grows as the distance between the sampling and constraint points.

Using the diffuse derivatives, the divergence of a vector field

$$
\nabla \cdot \mathbf{u}=\frac{\partial u}{\partial x}+\frac{\partial v}{\partial y}+\frac{\partial w}{\partial z}
$$

can be approximated as

$$
\nabla \cdot \mathbf{u} \simeq \frac{\partial \mathbf{b}^{T}}{\partial x} \mathbf{c}_{x}+\frac{\partial \mathbf{b}^{T}}{\partial y} \mathbf{c}_{y}+\frac{\partial \mathbf{b}^{T}}{\partial z} \mathbf{c}_{z} .
$$

We will use this equation in the next section to develop the divergence constraint for MLS.

\subsection{Divergence Constraint}

Huerta et al. [13] used the divergence constraint

$$
\nabla \cdot \mathbf{u}=\sigma
$$

to improve their description of sub-grid incompressibility with $\sigma=0$. We extend it to control divergence in velocity interpolation by allowing $\sigma$ to have non-zero values.

To add divergence constraints to $\mathbf{B}_{v e c}$, Equation (10) must be rewritten as

$$
\nabla \cdot \mathbf{u} \simeq \mathbf{b}_{d i v}^{T}(\mathbf{x}) \cdot \mathbf{c},
$$

where $\mathbf{b}_{d i v}$ is a vector in $\mathbb{R}^{k d}$, defined as follows:

$$
\begin{aligned}
& \mathbf{b}_{d i v}(\mathbf{x}) \\
& \left.=\frac{\partial\left[\mathbf{b}^{T}(\mathbf{x})\right.}{\partial x} \mathbf{0} \mathbf{0}\right] \\
& =\left[\begin{array}{lll}
\frac{\partial \mathbf{b}^{T}(\mathbf{x})}{\partial x} & \mathbf{0} & \mathbf{0}
\end{array}\right]+\left[\begin{array}{lll}
\mathbf{0} & \left.\frac{\partial \mathbf{b}^{T}(\mathbf{x})}{\partial y} \mathbf{0}\right] \\
\partial y & \mathbf{0}
\end{array}\right]+\left[\begin{array}{lll}
\mathbf{0} & \mathbf{0} & \frac{\partial \mathbf{b}^{T}(\mathbf{0})}{\partial z}
\end{array}\right] \\
& =\left[\begin{array}{lll}
\frac{\partial \mathbf{b}^{T}(\mathbf{x})}{\partial x} & \frac{\partial \mathbf{b}^{T}(\mathbf{x})}{\partial y} & \frac{\partial \mathbf{b}^{T}(\mathbf{x})}{\partial z}
\end{array}\right] .
\end{aligned}
$$

The partial derivatives of $\mathbf{b}$ are determined by differentiating each element of $\mathbf{b}$ separately. For example, if $d=3$ and $m=2$, then

$$
\begin{array}{r}
\frac{\partial \mathbf{b}(\mathbf{x})}{\partial x}=[0,1,0,0,2 x, 0,0, y, 0, z] \\
\frac{\partial \mathbf{b}(\mathbf{x})}{\partial y}=[0,0,1,0,0,2 y, 0, x, z, 0] \\
\frac{\partial \mathbf{b}(\mathbf{x})}{\partial z}=[0,0,0,1,0,0,2 z, 0, y, x] .
\end{array}
$$

By inserting the divergence constraint

$$
\mathbf{b}_{d i v}^{T} \cdot \mathbf{c}=\sigma
$$

into Equation (6), we obtain the divergenceconstrained version of MLS, which is

$$
\mathbf{W}_{d i v}(\mathbf{x}) \mathbf{B}_{d i v} \mathbf{c}_{v e c}(\mathbf{x})=\mathbf{W}_{d i v}(\mathbf{x}) \Phi_{d i v} .
$$

This equation can be solved using the method introduced in Section 2.

In practice, there are several ways to position the divergence constraints and thus to define $\mathbf{B}_{d i v}, \Phi_{d i v}$, and $\mathbf{W}_{d i v}$. A simple and efficient form is a single and moving divergence constraint

$$
\mathbf{W}_{d i v}=\left[\begin{array}{c}
\mathbf{W}_{v e c} \\
\omega_{d i v}
\end{array}\right], \mathbf{B}_{d i v}=\left[\begin{array}{c}
\mathbf{B} \\
\mathbf{b}_{d i v}
\end{array}\right],
$$

and $\Phi_{d i v}=[\Phi, \sigma] . \quad \mathbf{W}_{d i v}$ is a diagonal $(d N+$ $1) \times(d N+1)$ matrix, $\mathbf{B}_{d i v}$ is a $(d N+1) \times d k$ matrix, and $\Phi_{d i v}$ is a vector in $\mathbb{R}^{d N+1}$. Note the small increase in matrix sizes. This method of constraint positioning will be compared to some alternatives in the next section.

\section{Examples}

\subsection{Divergence-free Interpolations}

We performed several tests using divergence constraints positioned as shown in Figure 2(a). The vector field is defined on a unit square centered at $(0.5,0.5), \mathbf{p}_{1}$ is $(0.5,0.55), \mathbf{u}_{1}$ is $(0,-1)$, $\mathbf{p}_{2}$ is $(0.5,0.45)$, and $\mathbf{u}_{2}$ is $(0,1)$. More constraints of the form $\mathbf{u}_{i}=(0,0)$ are placed on the boundaries of the square with a spacing of 0.1 , making 38 constraints in total.

First, we compared naïve MLS vector implementation with the constrained technique due 
to Huerta et al. [13], who placed pseudodivergence-free constraints on all control points, resulting in the system

$$
\mathbf{W}_{p d f 1}=\left[\begin{array}{c}
\omega\left(\left|\mathbf{r}_{1}\right|\right) \\
\omega\left(\left|\mathbf{r}_{1}\right|\right) \\
\omega\left(\left|\mathbf{r}_{1}\right|\right) \\
\omega\left(\left|\mathbf{r}_{1}\right|\right) \\
\ddots \\
\omega\left(\left|\mathbf{r}_{N}\right|\right) \\
\omega\left(\left|\mathbf{r}_{N}\right|\right) \\
\omega\left(\left|\mathbf{r}_{N}\right|\right) \\
\omega\left(\left|\mathbf{r}_{N}\right|\right)
\end{array}\right]
$$

$$
\mathbf{B}_{p d f 1}=\left[\begin{array}{cccc}
\mathbf{b}\left(\mathbf{p}_{1}\right) & \mathbf{0} & \mathbf{0} \\
\mathbf{0} & \mathbf{b}\left(\mathbf{p}_{1}\right) & \mathbf{0} \\
\mathbf{0} & \mathbf{0} & \mathbf{b}\left(\mathbf{p}_{1}\right) \\
\mathbf{b}_{d i v}\left(\mathbf{p}_{1}\right) \\
\vdots \\
\mathbf{b}\left(\mathbf{p}_{N}\right) & \mathbf{0} & \mathbf{0} \\
\mathbf{0} & \mathbf{b}\left(\mathbf{p}_{N}\right) & \mathbf{0} \\
\mathbf{0} & \mathbf{0} & \mathbf{b}\left(\mathbf{p}_{N}\right) \\
\mathbf{b}_{d i v}\left(\mathbf{p}_{N}\right)
\end{array}\right]
$$

and $\Phi_{p d f 1}=\left[\mathbf{u}_{1}, 0, \cdots, \mathbf{u}_{N}, 0\right] . \quad \mathbf{W}_{p d f 1}$ is a diagonal $(d+1) N \times(d+1) N$ matrix, $\mathbf{B}_{p d f 1}$ is a $(d+1) N \times d k$ matrix, and $\Phi_{p d f 1}$ is a vector in $\mathbb{R}^{(d+1) N}$. Figures 2 and 3 show the vector interpolations with and without this type of divergence constraint. The divergence constraint allows the interpolated velocities to have non-zero horizontal components, even though the given constraints only have vertical components. This makes the whole velocity field nearly divergence-free, although there are local errors because of the diffusive derivative approximation: that is why we use the term 'pseudo-divergence-free'.

Next, we added an additional divergence constraint which moves with the position of evaluation $\mathbf{x}$, as follows:

$$
\mathbf{W}_{p d f 2}=\left[\begin{array}{c}
\mathbf{W}_{p d f} \\
\omega(0)
\end{array}\right]
$$

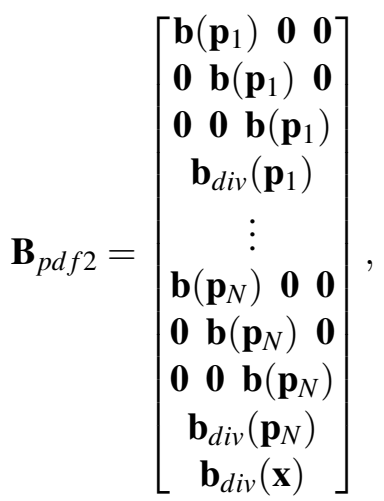

and $\Phi_{p d f 2}=\left[\Phi_{p d f 1}, 0\right] . \quad \mathbf{W}_{p d f 2}$ is a diagonal $((d N+1) N+1) \times((d+1) N+1)$ matrix, $\mathbf{B}_{p d f 2}$ is a $(d+1) N+1 \times d k$ matrix, and $\Phi_{p d f 2}$ is a vector in $\mathbb{R}^{(d+1) N+1}$. This improves the horizontal components of the interpolated vectors a little, which can be appreciated when Figure 4(b) is compared with Figure .

We then placed a single divergence constraint at the sampling position: this constraint moves with the sampling position. This compact form was proposed at the end of Section 3.2. We set $\sigma=0$ for this test. Comparing Figure 4(c) with Figure 4(b) shows that the result is essentially unchanged, but the matrix sizes are reduced and the implementation is simplified. Therefore, we used this formulation for all remaining examples.

Up to now, we have used the standard weight function $\omega(|\mathbf{r}|)=1 /\left(|\mathbf{r}|^{2}+\varepsilon^{2}\right)$, where $\varepsilon$ is a constant that controls the accuracy of the interpolation and we use $\varepsilon=0.0001$ for all examples. To obtain a closer approximation to divergencefree velocity field, we tried $\omega(|\mathbf{r}|)=1 /\left(|\mathbf{r}|^{4}+\right.$ $\left.\varepsilon^{2}\right)$ and $\omega_{d i v}=1 / \varepsilon^{2}$ instead of the standard weight functions. This produced a significant improvement as shown in Figure 5. There is a greater change in velocity direction which satisfies the divergence condition more fully, and allows more accurate momentum conservation.

\subsection{Divergence-free Extrapolation}

Divergence-free extrapolation was first proposed by Sussman [14] and introduced to computer graphics by Rasmussen et al. [15] to enhance the mass conservation property and smoothness of water surfaces. We performed a similar test to that of Rasmussen et al. [15]. Figure 6 , and the accompanying video, show two circular water bodies about to collide. The neg- 


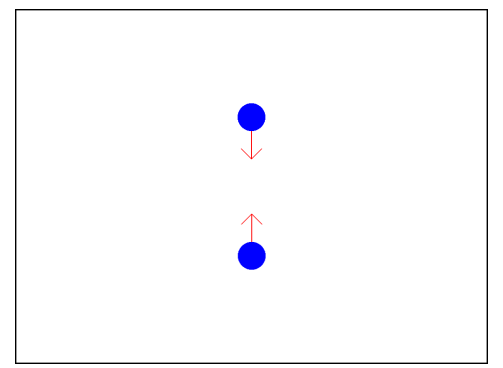

(a) Constraints

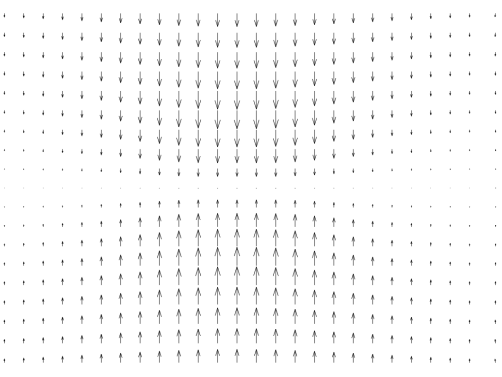

(b) MLS only (c) MLS with PDF constraints

Figure 2: Vector interpolation using MLS, with and without divergence constraints.

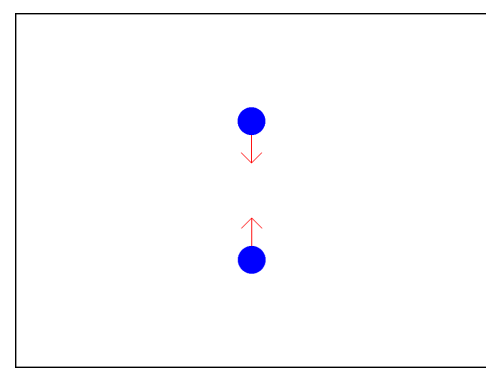

(a) Constraints

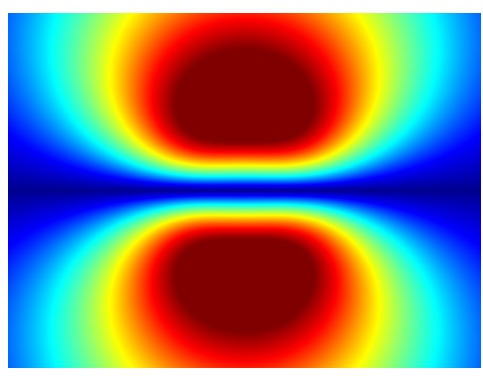

(b) MLS only

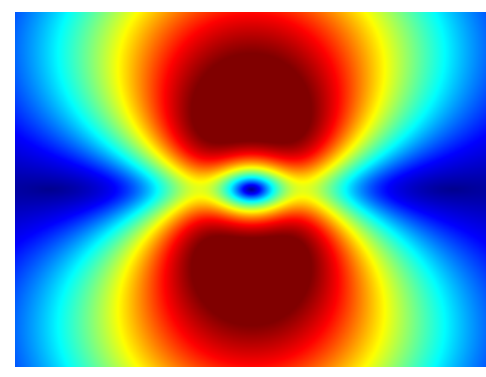

(c) MLS with PDF constraints

Figure 3: A version of Figure 2 colored by vector magnitude.

ative marker particles of the particle level set method $[16,17]$ naturally try to avoid each other. In this situation, our divergence-constrained MLS does not require the additional grid-scale projection step to make the extrapolated velocities divergence-free. This means that our method can be employed to improve subgrid details of fluid simulation.

\subsection{Divergence Control}

Divergence control has become popular in fluid simulation. For example, Feldman et al. [18] used it to model expanding fluids, and Kim et al. [19] used it to control bubble volumes. We can obtain similar effects by allowing a non-zero $\sigma$, as shown in Figure 7, without requiring a Poisson solver. In this example a total of 25 zero velocity constraints were used: one is placed at the center of a circle of radius 0.005 and the others are placed on its circumference.

\subsection{Fluid-like Particle Simulations}

Because we can control divergence, we can approximate the motion of fluids by animating control particles with a very simple advection and friction model, as follows:

$$
\begin{gathered}
\mathbf{p}_{i}^{n+1}=\mathbf{p}^{n}+\mathbf{u}_{i}^{n} \triangle t \\
\mathbf{u}_{i}^{n+1}=\mathbf{u}_{i}^{n}+\left(\mathbf{f}_{\text {friction }}+\mathbf{f}_{\text {gravity }}\right) \triangle t .
\end{gathered}
$$

We used a heuristic friction model based on forward differencing, very similar to

$$
\mathbf{f}_{\text {friction }}=k \frac{\partial \mathbf{u}^{n}}{\partial \frac{\mathbf{u}_{i}^{n}}{\left|\mathbf{u}_{i}^{n}\right|}}=\mathbf{u}_{i}^{n}+k \frac{\mathbf{u}^{n}\left(\mathbf{p}^{n+1}\right)-\mathbf{u}_{i}^{n}}{\left|\mathbf{u}_{i}^{n}\right|} .
$$

Results in 2D without gravity are given in Figure 8 and on the accompanying videos. The errors due to the diffusive-derivative assumption cause excessive particle gathering, but this effect could probably be reduced by a divergence control that would allow non-zero $\sigma$. There are 500 particles in each animation, which took 1 hours on a PC with an Intel Core2 CPU running at 3 GHz. A 3D example with gravity is shown in Figure 9 and on the accompanying videos. All of the fluid-like motions are created by velocity interpolation without any dynamics solver. This animation took 15 hours with a maximum of 756 moving particles and 600 boundary particles. We believe that code optimizations such as spatial localization could make this much faster. 


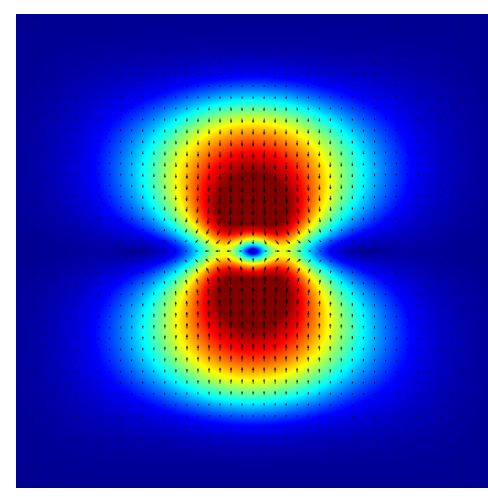

(a) Using $\mathbf{B}_{p d f 1}$

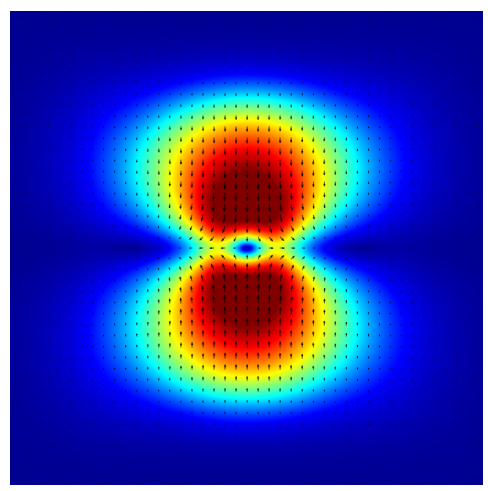

(b) Using $\mathbf{B}_{p d f 2}$

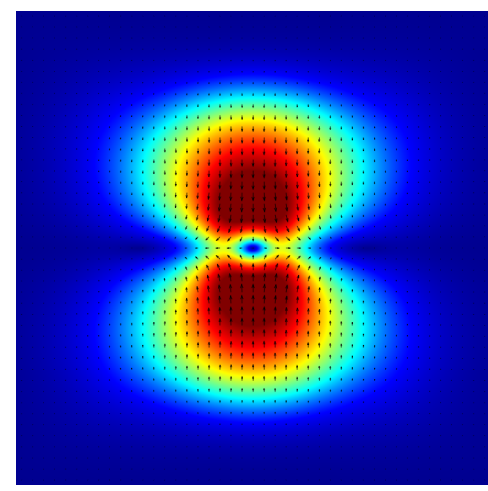

(c) Using $\mathbf{B}_{d i v}$

Figure 4: Various types of constraint: little difference is apparent between the results, but we adapted $\mathbf{B}_{\text {div }}$ because it is the simplest.

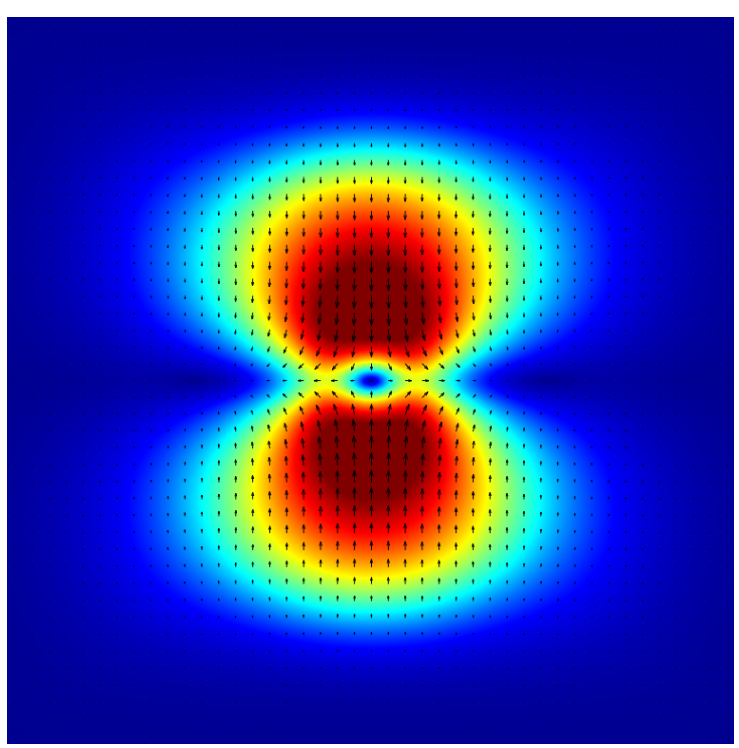

(a) $\omega=1 /\left(|\mathbf{r}|^{2}+\varepsilon^{2}\right)$

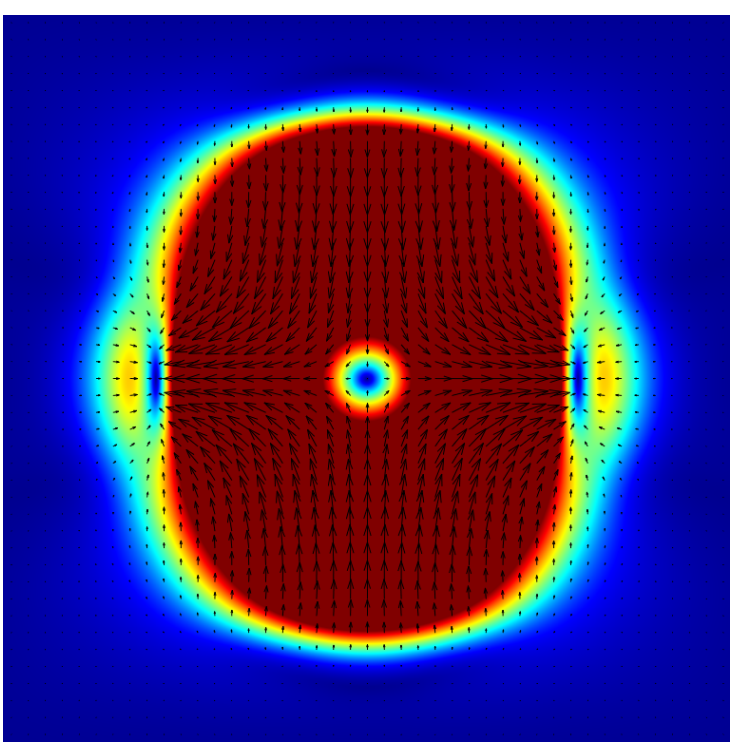

(b) $\omega=1 /\left(|\mathbf{r}|^{4}+\varepsilon^{2}\right)$

Figure 5: Improvement by higher degree weight function.

\section{Conclusion}

We have presented a divergence-constrained MLS method, based on the diffusive derivatives, to interpolate fluid velocities. It is shown that this method can be used for the divergence-free vector interpolation, divergence-free extrapolation, and to produce a divergence-controlled velocity field. We also extended the method to generate a fluid-like particle animation without using a fluids solver. This shows the potential of our approach in the generation of subgrid-scale divergence-free velocity fields.

A lot of further work is desirable. An application is to use this method as a plugin that al- lows existing fluid simulators to achieve better physical interpolation at subgrid scales. Developing a new CIP method [20] based on MLS or subgrid divergence-free extrapolation would also be straightforward as well, and it would be interesting to explore the possibility of using accurate derivative constraints instead of the diffusive derivatives. A fast GPU implementation ${ }^{1}$ would also be required to make this method popular. Finally, we are considering the use of discontinuous divergence-free interpolation of multi-phase fluids for modeling bubbles [21] and flames [22].

\footnotetext{
${ }^{1}$ http://www.nvidia.com/object/cuda_home.html
} 


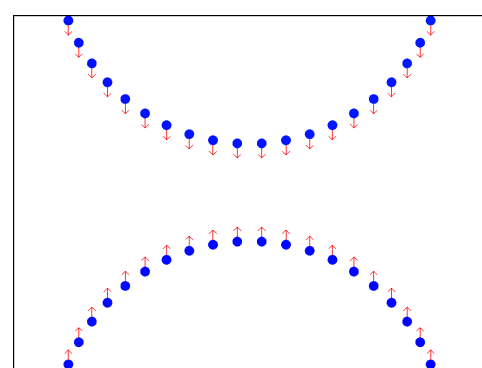

(a) Given constraints

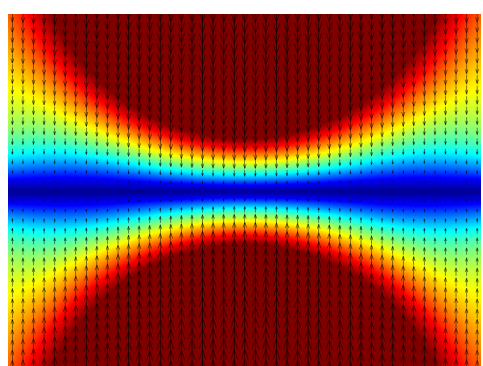

(b) Naïve velocity extrapolation

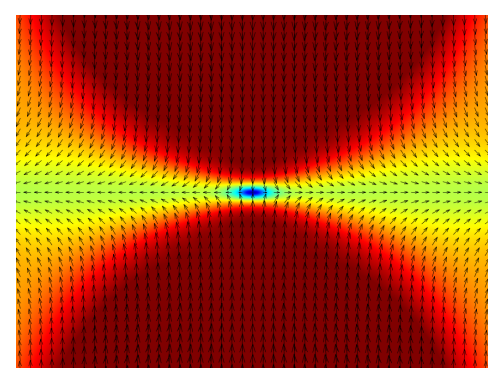

(c) Divergence-free velocity extrapolation

Figure 6: Divergence-free velocity extrapolation using MLS. The accompanying video shows massless particles moving in these velocity fields.

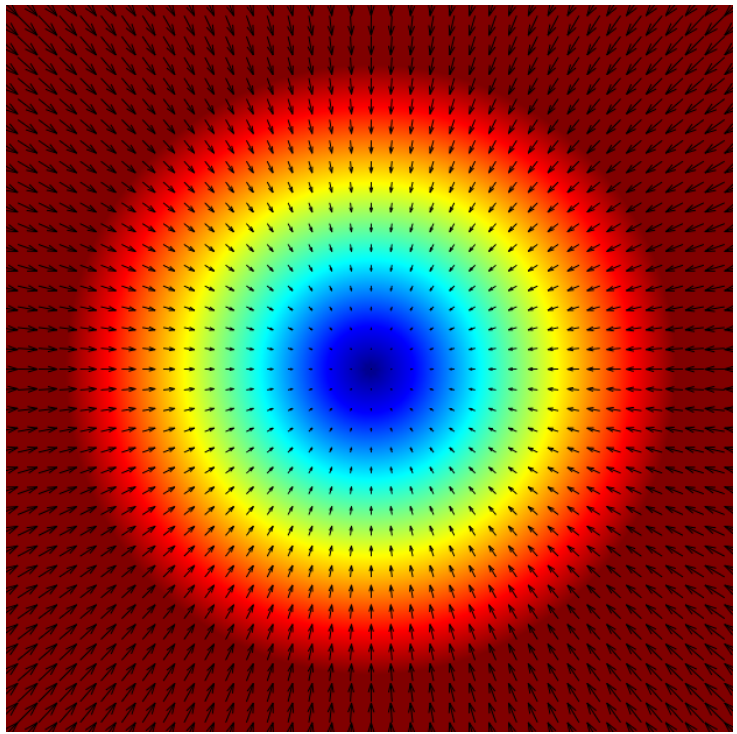

(a) $\sigma=-10$,

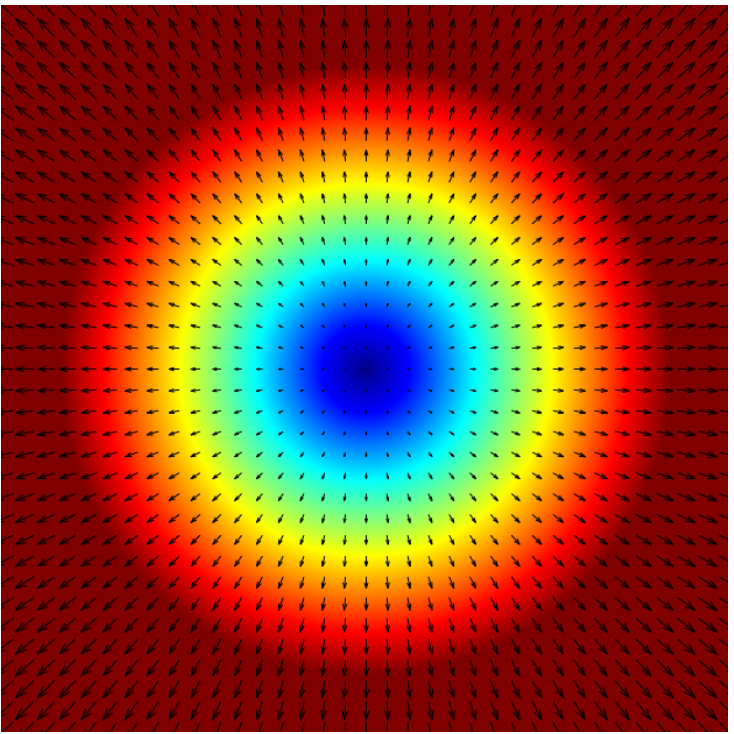

(b) $\sigma=10$

Figure 7: Divergence-controlled velocity fields.

\section{Acknowledgements}

\section{References}

[1] Jos Stam. Stable fluids. In Proc. of SIGGRAPH 99, pages 121-128, 1999.

[2] B. Kim, Y. Liu, I. Llamas, and J. Rossignac. Advections with significantly reduced dissipation and diffusion. IEEE Trans. on Vis. and Comput. Graph., 13:135-144, 2007.

[3] O. Song, Hyuncheol Shin, and HyeongSeok Ko. Stable but non-dissipative water. ACM Trans. Graph., 24(1):81-97, 2005.

[4] Janghee Kim, Deukhyun Cha, Byungjoon
Chang, Bonki Koo, and Insung Ihm. Practical animation of turbulent splashing water. In Proceedings of the 2006 ACM SIGGRAPH/Eurographics Symp. on Comput. Anim., pages 335-344, 2006.

[5] F. Losasso, J. Talton, N. Kwatra, and R. Fedkiw. Two-way coupled SPH and particle level set fluid simulation. IEEE Trans. on Vis. and Comput. Graph. (In Press), 2008.

[6] J.-M. Hong, H.-Y. Lee, J.-C. Yoon, and C.-H. Kim. Bubbles alive. ACM Trans. Graph. (SIGGRAPH Proc.), To Appear, 2008.

[7] B. Feldman, J. O'Brien, and B. Klingner. Animating gases with hybrid meshes. 


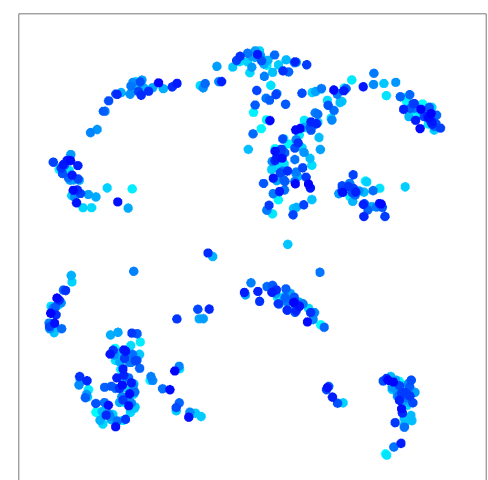

(a) $\sigma=0$

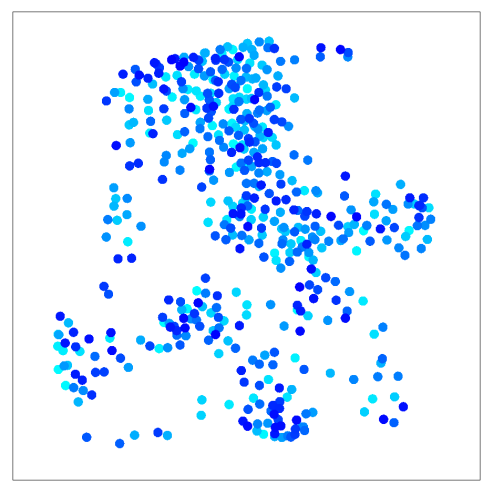

(b) $\sigma=10$

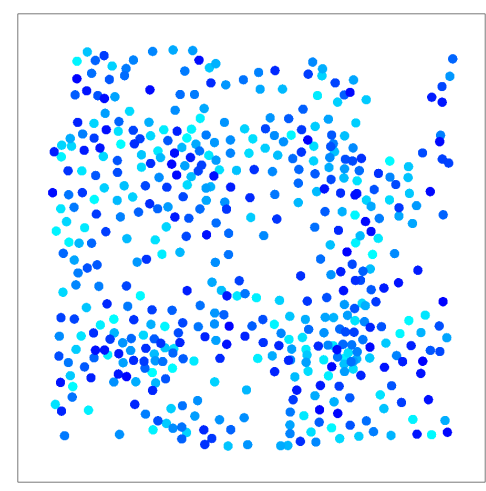

(c) $\sigma=100$

Figure 8: Fluid-like particle animations in 2D, achieved without a fluid dynamics solver.

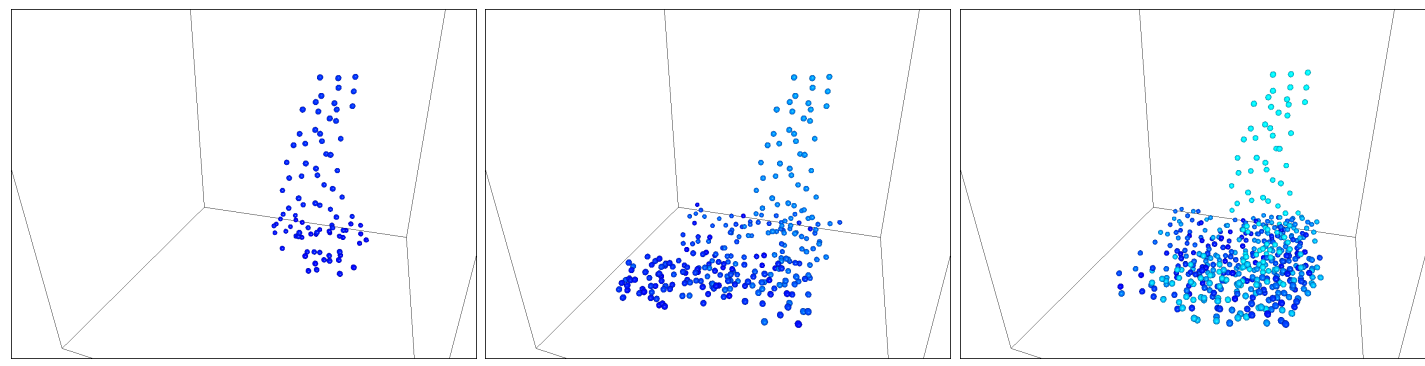

Figure 9: Fluid-like particle animation in 3D with gravity.

ACM Trans. Graph. (SIGGRAPH Proc.), 24(3):904-909, 2005.

[8] C. Shen, J. F. O'Brien, and J.R. Shewchuk. Interpolating and approximating implicit surfaces from polygon soup. In $A C M$ Trans. Graph. (SIGGRAPH Proc.), volume 31, pages 321-328, 2004.

[9] T. Belytschko, Y. Krongauz, D. Organ, M. Fleming, and P. Krysl. Meshless methods: An overview and recent developments. Comput. Methods Appl. Mech. Engrg., 139:3-47, 1996.

[10] M. Müller, R. Keiser, A. Nealen, M. Pauly, M. Gross, and M. Alexa. Point based animation of elastic, plastic and melting objects. In Proc. of the 2004 ACM SIGGRAPH/Eurographics Symp. on Comput. Anim., pages 141-151, 2004.

[11] A. Nealen. An as-short-as possible introduction to the least squares, weighted least squares and moving least squares methods for scattered data approximation and inter- polation. Technical report, TU Darmstadt, 2004.

[12] B. Nayroles, G. Touzot, and P. Villon. Generalizing the finite element method: Diffuse approximation and diffuse elements. Computational Mechanics, 10:307-318, 1992.

[13] A. Huerta, Y. Vidal, and P. Villon. Pseudodivergence-free element free galerkin method for incompressible fluid flow. Comput. Methods Appl. Mech. Engrg., 193:1119-1136, 2004.

[14] M. Sussman. A second order coupled level set and volume-of-fluid method for computing growth and collapse of vapor bubbles. J. Comput. Phys., 187:110-136, 2003.

[15] N. Rasmussen, D. Enright, D. Nguyen, S. Marino, N. Sumner, W. Geiger, S. Hoon, and R. Fedkiw. Directable photorealistic liquids. In Proc. of the 2004 ACM SIGGRAPH/Eurographics Symp. on Comput. Anim., pages 193-202, 2004. 
[16] D. Enright, D. Nguyen, F. Gibou, and R. Fedkiw. Using the particle level set method and a second order accurate pressure boundary condition for free surface flows. In Proc. 4th ASME-JSME Joint Fluids Eng. Conf., number FEDSM200345144. ASME, 2003.

[17] D. Enright, S. Marschner, and R. Fedkiw. Animation and rendering of complex water surfaces. ACM Trans. Graph. (SIGGRAPH Proc.), 21(3):736-744, 2002.

[18] Bryan E. Feldman, James F. O'Brien, and Okan Arikan. Animating suspended particle explosions. ACM Trans. Graph. (SIGGRAPH Proc.), 22(3):708-715, 2003.

[19] Byungmoon Kim, Yingjie Liu, Ignacio Llamas, Xiangmin Jiao, and Jarek Rossignac. Simulation of bubbles in foam with the volume control method. ACM Trans. Graph. (SIGGRAPH Proc.), 26(3):481-487, 2007.

[20] T. Yabe, F. Xiao, and T. Utsumi. Constrained interpolation profile method for multiphase analysis. J. Comput. Phys., 169:556-593, 2001.

[21] J.-M. Hong and C.-H. Kim. Discontinuous fluids. ACM Trans. Graph. (SIGGRAPH Proc.), 24(3):915-920, 2005.

[22] J.-M. Hong, T. Shinar, and Ron Fedkiw. Wrinkled flames and cellular patterns. ACM Trans. Graph. (SIGGRAPH Proc.), 26(3):471-476, 2007. 